\title{
3-dimensional modelling of the DOEs formation in PVA/AA photopolymers
}

Fernández, R., Gallego, S., Márquez, A., Francés, J., Neipp, C., et al.

R. Fernández, S. Gallego, A. Márquez, J. Francés, C. Neipp, D. Puerto, E. M. Calzado, I. Pascual, A. Beléndez, "3-dimensional modelling of the DOEs formation in PVA/AA photopolymers," Proc. SPIE 11367, Photosensitive Materials and their Applications, 113671E (1 April 2020); doi: $10.1117 / 12.2555692$

SPIE. Event: SPIE Photonics Europe, 2020, Online Only, France 


\title{
3-Dimensional modelling of the DOEs formation in PVA/AA photopolymers
}

\author{
R. Fernández ${ }^{\mathrm{a}}$, S. Gallego ${ }^{\text {a, b }}$, A. Márquez ${ }^{\mathrm{a}, \mathrm{b}}$, J. Francés ${ }^{\mathrm{a}, \mathrm{b}}$, C. Neipp ${ }^{\mathrm{a}, \mathrm{b}}$, D. Puerto ${ }^{\mathrm{a}, \mathrm{b}}$, E. M. \\ Calzado $^{\text {a, b }}$, I. Pascual ${ }^{\text {a, c }}$, A. Beléndez ${ }^{\text {a, b }}$ \\ ${ }^{a}$ I.U. Fisica Aplicada A Las Ciencias Y Las Tecnologíasm University of Alicante, Spain, Apartat 99 \\ E-03080; ${ }^{b}$ Dept. Física, Enginyeria de Sistemes i Teoria del Senyal, Universitat d'Alacant, Spain; \\ ${ }^{\mathrm{c}}$ Dept. Óptica, Farmacologia i Anatomia, Universitat d'Alacant (Spain)
}

\begin{abstract}
Photosensitive materials and optical information processing technologies based on holographic and photonic techniques are suffering a huge improvement. Furthermore Spatial Light Modulators (SLMs) based on LCoS microdisplays (PA$\mathrm{LCOS}$ ) open new possibilities to modulate the wavefront of a light beam.

The improving of the models of the photopolymers as optical recording material together with the modelling of PALCoS, high resolution reflective devices, make possible the generation and recording of Diffractive Optical Elements (DOE) on the photosensitive materials. This DOEs have many important applications in photonics, communications of optical information processing.

Working with a setup based on a LCoS display as a master, we can store complex DOEs. We used in this work PVA/AA based on acrylamide with coverplating and index matching system to avoid the influence of the thickness variation on the transmitted light in the material.

With the 3-Dimensional diffusion model we can predict the DOE properties before recording and optimize the recording time and the exposure dose. Experimental data is compared with the simulation results to evaluate the accuracy of our model to reproduce the recording of any kind of complex DOE onto a photopolymer. This allows us to choose the appropriate characteristics for the material depending on the application and evaluate the influence of different parameters involved in the DOE generation.

In this work we evaluate the simulation of the recording of optical vortexes, axicons, fork gratings and diffractive lenses comparing with the results using our experimental set-up.
\end{abstract}

Keywords: Diffractive elements, optical recording materials, Spatial Light Modulators

\section{INTRODUCTION}

The demand for competitive and more complex DOE is growing each year [1]. Spatial light modulations technology can supply in part this demand, the pixel size has been reduced to few microns, nevertheless the pixel crosstalk, also called the fringing field effect, which causes the response of these devices to deviate from the ideal behavior and its elevate price are important drawbacks for the massive production.

On the other hand, the potential of photopolymers to fabricate DOE has been demonstrated by many authors using different chemical compositions [2-4]. The characterization of these materials was at the beginning experimental [5], later diffusion models were introduced to study the generation of the holographic sinusoidal gratings. The models were become more sophisticated with the passing of the years [6-11]. Some interesting effects were introduced in these models such as: non-local polymerization, variations of the polymerization rate, the light attenuation with depth, etc. [611]. But generally the models were applied to the one sinusoidal grating formation created by the interference of two planar waves.

In order to use photopolymers for a wide range of applications high accuracy of the diffusion models is required, even when the DOE are complex. In this sense, the photopolymers based on PVA/AA presented a large discussion to fit the

Photosensitive Materials and their Applications, edited by Robert R. McLeod, Inmaculada Pascual Villalobos,

Yasuo Tomita, John T. Sheridan, Proc. of SPIE Vol. 11367, 113671E · C 2020 SPIE

CCC code: $0277-786 \mathrm{X} / 20 / \$ 21 \cdot$ doi: $10.1117 / 12.2555692$ 
monomer diffusion in this kind of materials [12]. Once the basic recording parameters that govern the DOE formation in the material are known a 3-Dimensial model can be proposed to store complex DOEs [13].

In general the DOE formation depends on the polymerization rate, $F_{R}$, and the molecules diffusion inside the material, $\mathrm{D}_{\mathrm{m}}$, due to the Fick's Law. In particular for PVA/AA materials the effects due to the surface variations can be eliminated using coverplating and refractive index matching.

Then the differential equations for the monomer volume fraction, $\mathrm{M}$ and polymer volume fraction, $\mathrm{P}$, can be written as follows:

$$
\begin{aligned}
& \frac{\partial M(x, y, z, t)}{\partial t}=\frac{\partial}{\partial z} D_{m}(t) \frac{\partial M(x, y, z, t)}{\partial z}+\frac{\partial}{\partial y} D_{m}(t) \frac{\partial M(x, y, z, t)}{\partial y} \\
& +\frac{\partial}{\partial x} D_{m}(t) \frac{\partial M(x, y, z, t)}{\partial x}-F_{R}(x, z, t) M(x, z, t) \\
& \frac{\partial P(x, y, z, t)}{\partial t}=F_{R}(x, y, z, t) M(x, y, z, t)
\end{aligned}
$$

The polymerization rate, $\mathrm{F}_{\mathrm{R}}$, depends on the reaction kinetics and the recording intensity, $\mathrm{I}$; this dependence can be described by the following equation:

$$
F_{R}(x, y, z, t)=k_{R}(x, y, z, t) I(x, y, z, t)^{\gamma}=k_{R}(x, y, z, t) I(x, y)^{\gamma} e^{-\alpha(t) \gamma}
$$

Where $\mathrm{I}$ is the recording intensity, $\mathrm{k}$ is the polymerization velocity, $\gamma$ indicates the relationship between intensity and polymerization rate, $0.96 ; \alpha$ is the coefficient of light attenuation. The initial value of $\alpha[\alpha(\tau=0)=\alpha 0]$ can be obtained if the transmittance and the physical thickness of the layer are known.

There are different methods to solve these differential equations. In this paper we use the finite-difference method (FDM) to solve a 3-dimensional problem using a rigorous method. Therefore, Eqs. (1) and (2) can be written as:

$$
\begin{aligned}
& M_{i, j, k}=\frac{\Delta t}{\Delta x^{2}} D_{m} M_{i+1, j, k-1}-2 \frac{\Delta t}{\Delta x^{2}} D_{m}(t) M_{i, j, k-1}+\frac{\Delta t}{\Delta x^{2}} D_{m}(t) M_{i-1, j, k-1}+\frac{\Delta t}{\Delta z^{2}} D_{m}(t) M_{i, j+1, k-1}-\frac{2 \Delta t}{\Delta z^{2}} D_{m}(t) M_{i, j, k-1}+ \\
& \frac{\Delta t}{\Delta z^{2}} D_{m}(t) M_{i, j-1, k-1}-\Delta t F_{R_{i, j, k-1}} M_{i, j, k .1}+M_{i, j, k-1} \\
& P_{i, j, k}=P_{i, j, k}+\Delta t * F_{R_{i, j, k-1}} * M_{i, j, k-1}
\end{aligned}
$$

For the spherical lenses, the Eqs. (8) and (9) are the following:

$$
\begin{aligned}
& M_{i, l, j, k}= \\
& \frac{\Delta t}{\Delta x^{2}} D_{m} M_{i+1, l, j, k-1}-2 \frac{\Delta t}{\Delta x^{2}} D_{m}(t) M_{i, l, j, k-1}+\frac{\Delta t}{\Delta x^{2}} D_{m}(t) M_{i-1, l, j, k-1}+\frac{\Delta t}{\Delta y^{2}} D_{m}(t) M_{i, l+1, j, k-1}-2 \frac{\Delta t}{\Delta y^{2}} D_{m}(t) M_{i, l, j, k-1}+ \\
& \frac{\Delta \mathrm{t}}{\Delta y^{2}} D_{m}(t) M_{i, l-1, j, k-1}+\frac{\Delta t}{\Delta z^{2}} D_{m}(t) M_{i, l, j+1, k-1}-2 \frac{\Delta t}{\Delta z^{2}} D_{m}(t) M_{i, l, j, k-1}+\frac{\Delta t}{\Delta z^{2}} D_{m}(t) M_{i, l, j-1, k-1}=\Delta t F_{R_{i, l, j, k-1}} M_{i, l, j, k .1}+ \\
& M_{i, l, j, k-1}
\end{aligned}
$$




$$
F_{R i, l, j, k}=F_{R i, l, j, k-1}+\Delta t F_{R i, l, j, k-1} M_{i, l, j, k-1}+M_{i, l, j, k-1}
$$

In order to guarantee the numerical stability of the Eqs., the increment in the time domain, $\Delta t$, must satisfy the stability criterion:

$$
\Delta \mathrm{t}<\frac{1}{2}\left(\frac{\Delta \mathrm{x}^{2}}{\mathrm{D}_{\mathrm{m}}}\right)
$$

In this case, we choose $\Delta t=0.1\left(\Delta x^{2} / D_{m}\right)$

Once we obtain the monomer and polymer concentrations, we can use the refractive index values to calculate the refractive index distribution during the recording process. The refractive index distribution can be measured using the Lorenz-Lorenz Eq. as follows:

$$
\frac{n^{2}-1}{n^{2}+2}=\frac{n_{m}^{2}-1}{n_{m}^{2}+2} M+\frac{n_{p}^{2}-1}{n_{p}^{2}+2} P+\frac{n_{b}^{2}-1}{n_{b}^{2}+2}\left(1-M_{0}\right)
$$

Where $\mathrm{M}_{0}$ is the average initial value for the volume fraction of monomer, $\mathrm{n}_{\mathrm{p}}$ is the polymer refractive index, $\mathrm{n}_{\mathrm{m}}$ is the monomer refractive index, and $\mathrm{nb}$ is the binder refractive index. The last two parameters can be measured using a refractometer and the value of $\mathrm{np}$ can be obtained using the zero spatial frequency technique [14].

In order to simulate the recording of the lenses in the photopolymer, the values of the main parameters are obtained from interferometric and diffractive techniques [13-14]. In this work these parameters are: $\mathrm{k}_{\mathrm{R}}=0.007 \mathrm{~cm}^{2} /(\mathrm{s} \cdot \mathrm{mW}), \alpha=0.02 \mu \mathrm{m}^{-}$

${ }^{1}, \mathrm{M}_{0}$ (volume fraction) $=0.22, \mathrm{I}_{0}=0.5 \mathrm{~mW} / \mathrm{cm}^{2}, \gamma$ (relationship between intensity and polymerization rate) $=0.93$ and $\mathrm{D}_{0}=3 * 10^{-10} \mathrm{~cm}^{2} / \mathrm{s}$.

\section{RESULTS}

In this section, some important aspects concerning the formation of a DOE on photopolymer using a LCOS as a master are discussed and analyzed. First, we describe the fitting of the main parameters introduced in the 3-D model, the polymerization rate, using zero spatial frequency limit recording and the monomer diffusion inside the material, using index matching and recording very low spatial frequency sinusoidal gratings. The analysis of sinusoidal gratings is the first step to check the validity of the model simulations. The next step is the simulation of more complex DOE: Lenses, axicons, and optical vortex.

\subsection{Parameters estimation}

As mentioned in the introduction, we previously measured all the parameters introduced in the diffusion model by means of metrology experiments based on interferometric and diffractive techniques [14]. One of the key parameters is the polymerization rate, which is measured by zero spatial frequency experiments. In Figure 1 we present the experimental results obtained for the standard composition optimized for diffractive lens recording [3]. Figure 1.a shows the variation in phase shift proportional to the movement of the interference fringes on the CCD camera at zero spatial frequency limit, where we can fit the polymerization rate taking into account the Trommsdorff effect. Furthermore, in Figure 1.b the low spatial sinusoidal gratings recording, during 50s, and the dark evolution to fit the monomer diffusion inside the material when polymerization do not take place [14]. In this figure, we compared the results in the same layer with index matching and the spatial period of the grating is $168 \mu \mathrm{m}$. As it can be seen, the experimental results are very close to the theoretical predictions and we obtain the initial value of the polymerization rate for this composition. In this material is also important fit the variation of the absorption as a function of energy dose, the model used is a 3-D model then the 
gradient of recording light inside material produce significant effects [15]. The materials can be prepared for a range of thickness between 30 and $600 \mu \mathrm{m}$, as thick is the layer more important are the depth attenuation effects.


Figure. 3.- Phase shift at zero spatial frequency recording as a function of exposure time and very low spatial frequency gratings recorded to fit the monomer diffusion in coverplated PVA/AA photopolymer.

\subsection{Modelling DOE}

We introduced diferent intesity pattens in the spatial light motulator working in amplitude mode. On the other and we simulate the formation of these DOEs with our model, that provide us a refractive index distribution in 3-D. To compare our model with the experiments we use the Fresnel propagation to obtain the focal point intensity distribution, in the case of difractive lenses or the far-field diffraction patterns for diffractive axicons, or others DOEs. In the experiments, we place the CD camera behind the recorded phase diffractive object, just at the point where we have made the Fresnel propagation. In general we obtain the far-field, but in the particular case of diffractive lenses que place the camera at the 
main focal point. In Figure 1 we present the first three DOEs that we want to simulate. Figure 2.a is a fork grating. This kind of gratings and diffractive axicons are used to generate Bessel beams characterized by a dark central core which propagates in free space without any spreading due to diffraction. Recently, optical beams that rotate around the optical axis are becoming an important focus of attention. Figure 2.b is a diffractive axicon definded as an element which forms a focal line along the optical axis, instead of focusing it in a point, normaly used to generate Besel beams. Figure $1 . \mathrm{c}$ is an helicoidal axicon. It is hybrid DOE formed by the combination of an axicon and a spiral phase plate(SPP). The latter is used to generate ring-patterned beams with helical wavefronts. Thus, by using a composite element that has the functions of both the spiral phase plate and axicon, a High-Order Bessel Beam can be generated in just one pass.

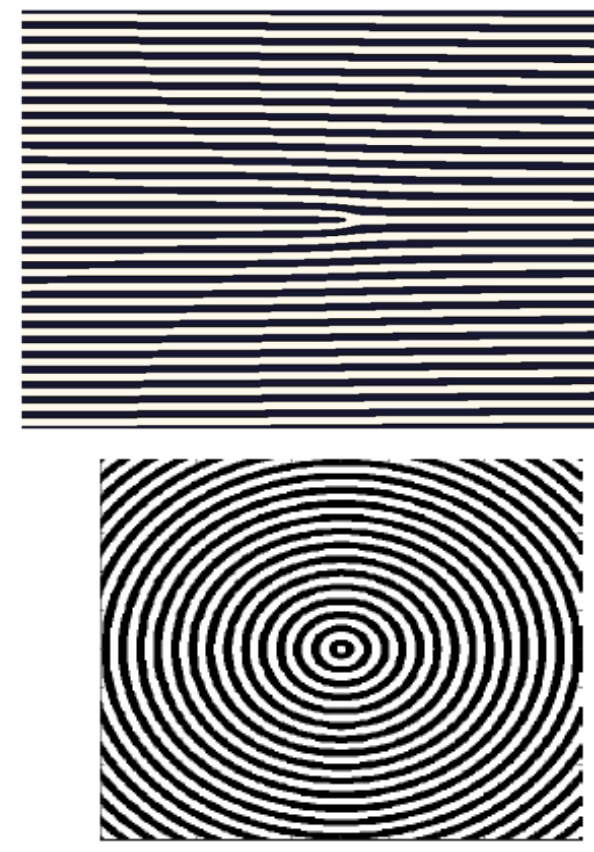

a)

b)

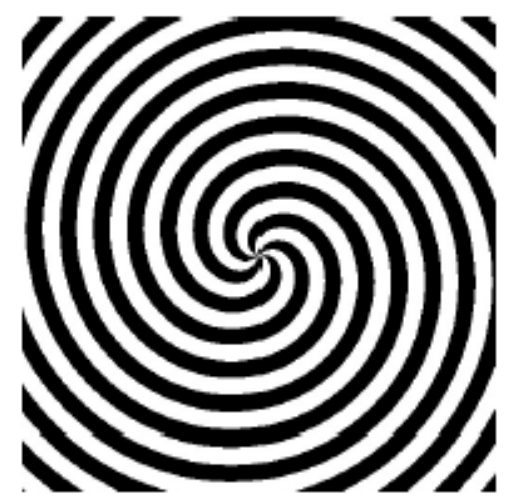

c)

Figure 2. a) Fork grating, b) diffractive axicon and c) helicoidal axicon introduced in the 3-D diffusion model and in the LCoS, working in amplitude mode to generate the appropriate intensity distribution.

There are different ways to explore and check the agreement between theoretical predictions reported by 3 -d model and experimental results. The fist way is more quantitative, and consists on the 2-D representation of the intensity versus distance normalized by the maximum value, it depend on the incident beam. An example is the Figure 3. In this figure the intensity distribution along a vertical line is depicted and we can see the good agreement between experimental and theoretical points. Nevertheless, the resolution of the experimental data is limited by optical properties of our experimental set-up, and the resolution of our CCD camera. In our case the pixel size of the camera used is around $7 \mu \mathrm{m}$. 
In opposition, for the simulation we can use the desired resolution increasing the calculation time of the program. The second way to analyse the agreement between experiments and simulations is more qualitative, is just, representing the far field diffraction pattern by 2-D image, like we can see in the CCD camera. Figure 4 and 5 show this agreement for the two analysed axicons. In the case of the helicoidal axicon, Figure 4, the experimental results are close to the theoretical simulations, but the definition of the ring was a little bit worse than the standard diffractive axicon (Figure 4).



Figure 3. Far field diffraction pattern for a fork grating. Obtained by 3-D model, continues line, and experimental data, dashed line.

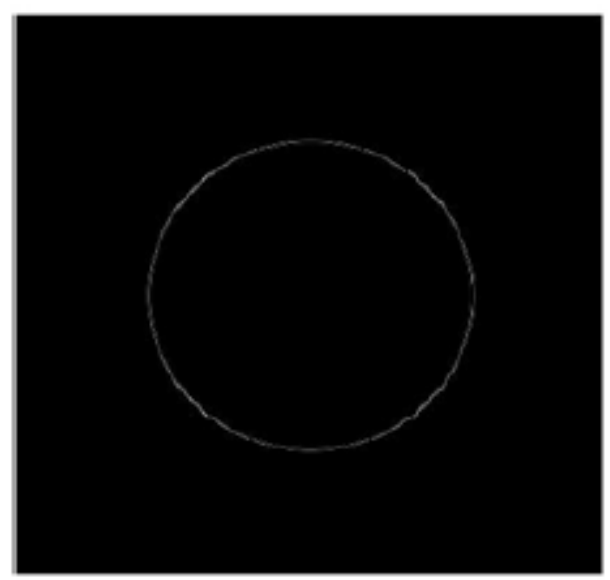

a)

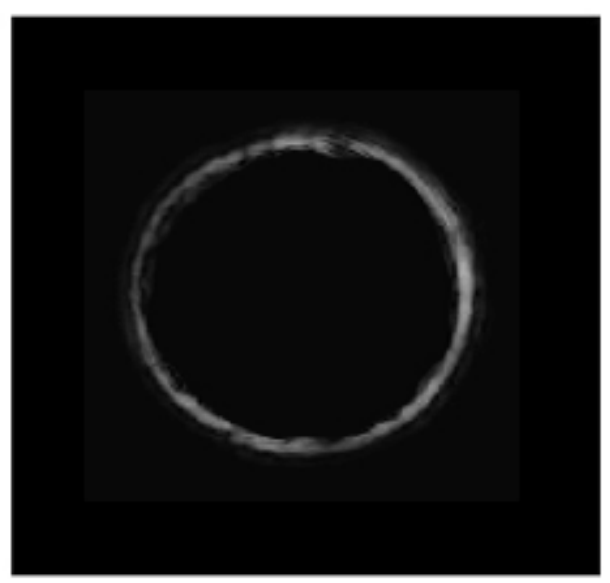

b)

Figure 4. Far field diffraction patern for a difractive axicon. A) Obtained by 3-D model. B) Experimental. 




Figure 5. Far field diffraction patern for a difractive Helicoidal axicon. A) Obtained by 3-D model. B) Experimental.

The using of the LCoS as a master, provide us a wide range of flexibility to record DOEs. One of the DOEs widely used is diffractive lens. In this case our experimental set-up give us the possibility to record lenses with different focal length without change any mechanical part of the set-up, we just modify the signal sent to the SLM. The desired intensity distribution to record a diffractive lens is represented in Figure 6. The comparison between the model prediction and the experiments is presented in Figure 7 for a lens with $20 \mathrm{~cm}$ of focal length. The accurate prediction for the diffractive lenses is clearly seen.

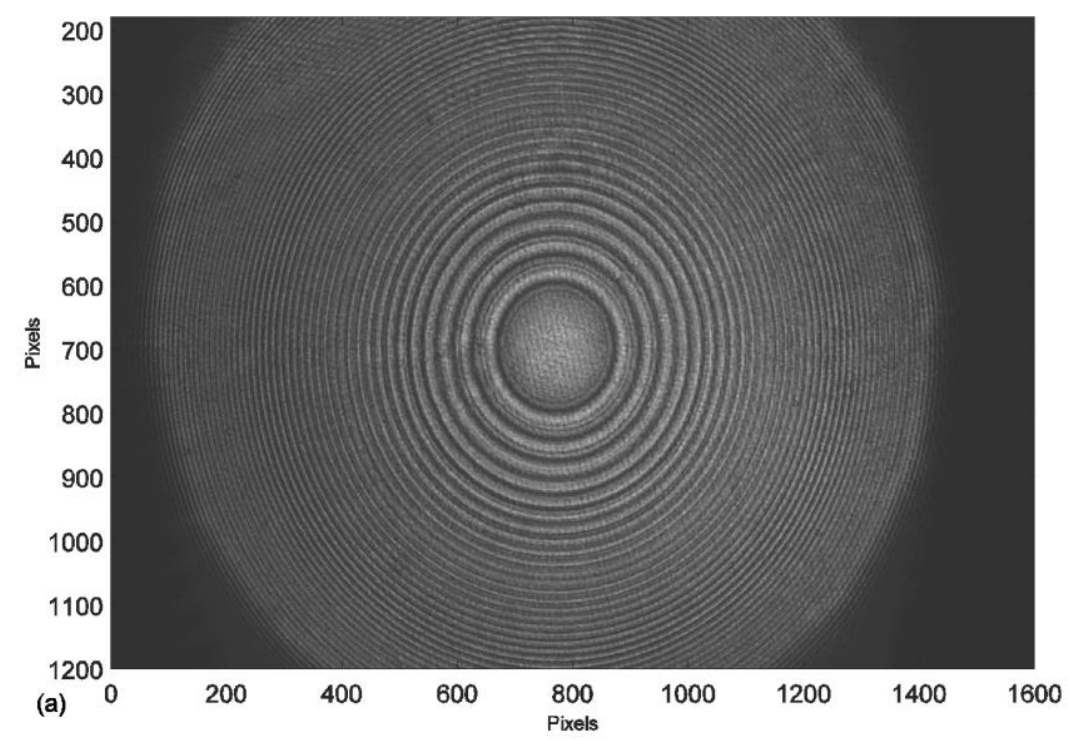

Figure 6. Diffractive lens pattern. 


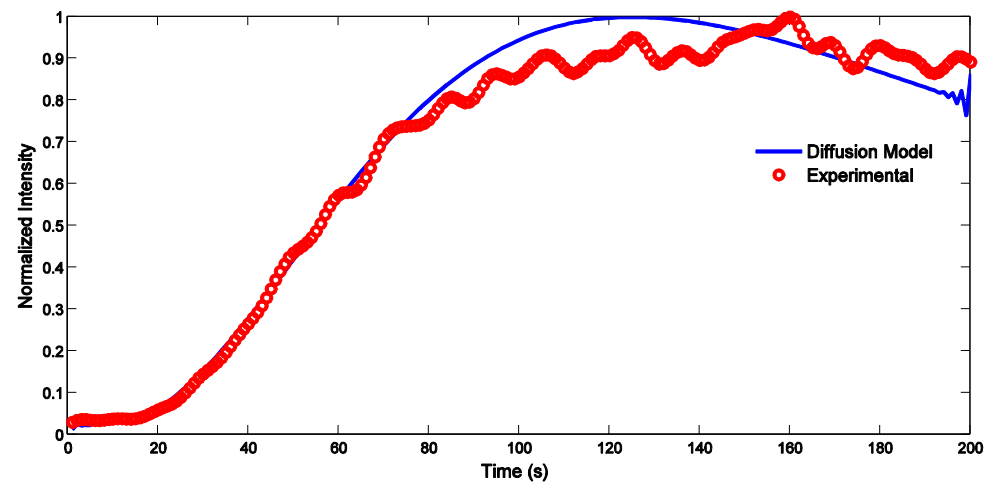

Figure 7. Intensity at the expected focal point of the diffractive lens. Experimental and simulated by 3-D model.

\section{CONCLUSIONS}

In this paper, we evaluated the suitability of our diffusion model to simulate the recording of different complex DOEs, such as fork gratings, diffractive and helicoidal axicons, diffractive lenses in a PVA/AA-based photopolymer. To validate the results obtained through the model, we recorded experimentally these complex DOEs thanks to a setup based on a LCOS SLM. This set up let us change the DOE stored without the movement of any mechanical part. Therefore we can easily test multiple DOE schemes. The results obtained showed the capacities of the 3-Dimensional diffusion model to predict the behavior of the material during the recording of these complex DOEs. This is particularly relevant as the selection of the material is one of the key aspects to obtain the desired results. In this sense, it is worth noting also the good response of the PVA/AA photopolymer storing the complex schemes evaluated, similar to the one exhibited for simpler DOEs as a gratings with different profiles.

Acknowledgments: This work was supported by The Ministerio de Ciencia, Innovación y Universidades (Spain) under project FIS2017-82919-R/(MINECO/AE/FEDER, UE). and FIS2015-66570-P (MINECO/FEDER). R.F. acknowledges the financial support by the Generalitat Valenciana (Spain) under grants APOSTD/2018/.

\section{REFERENCES}

[1] O’Shea, D. C., Suleski, T. J., Kathman, A. D. \& Prather, D. W. Diffractive Optics: Design, Fabrication, and Test (SPIE Press, Bellingham, WA, USA, (2004).

[2] Infusino, M.; Luca, A.D.; Barna, V.; Caputo, R.; Umeton, C. "Periodic and aperiodic liquid crystal-polymer composite structures realized via spatial light modulator direct holography," Opt. Express 20, 23138-23143 (2012).

[3] Fernández, R.; Gallego, S.; Márquez, A.; Francés, J.; Navarro-Fuster, V.; Pascual, I. "Diffractive lenses recorded in absorbent Photopolymers," Opt. Express 24, 1559-1572 (2016).

[4] Fernández, R.; Navarro-Fuster, V.; Martínez, F.; Gallego, S.; Márquez, A.; Pascual, I.; Beléndez, A. "Modeling diffractive lenses recording in environmentally friendly photopolymer. Polymers," 9, 278 (2017).

[5] Calixto, S. "Dry polymer for holographic recording," Applied Optics 26, 3904-3909 (1987).

[6] Zhao G. and Mouroulis P., "Diffusion model of hologram formation in dry photopolymers materials," J. Mod. Opt. 41, 1929-1939 (1994).

[7] Lougnot J., Jost P., and Lavielle L., "Polymers for holographic recording: VI. Some Basic ideas for modelling the Kinetics of the recording process," Pure and Appl. Opt. 6, 225-245 (1997).

[8] Piazzolla S. and Jenkins B. K., "First-harmonic diffusion model for holographic grating formation in photopolymers," J. Opt. Soc. Am. B 17, 1147-1157 (2000).

[9] Sheridan J. T. and Lawrence J. R., "Nonlocal-response diffusion model of holographic recording in photopolymer," J. Opt. Soc. Am. A 17, 1008-1014 (2000). 
[10] Moreau V., Renotte Y., and Lion Y., "Characterization of DuPont photopolymer: determination of kinetic parameters in a diffusion model," Applied Optics 41, 3427-3435 (2002).

[11] Gallego S., Ortuño M., Neipp C., Márquez A., Beléndez A., Pascual I., Kelly J. V., and Sheridan J. T., "3 Dimensional analysis of holographic photopolymers based memories," Opt. Express 13, 3543-3557 (2005).

[12] Gallego S., Fernández R., Márquez A., Ortuño M., Neipp C., Gleeson M. R., Sheridan J. T., and Beléndez A., "Two diffusion photopolymer for sharp diffractive optical elements recording," Opt. Lett. 40, 3221-3224 (2015).

[13]Fernández R, Gallego S, Márquez A, Neipp C, Calzado EM, Francés J, Morales-Vidal M, Beléndez A. "Complex Diffractive Optical Elements Stored in Photopolymers," Polymers 11(12) 1920 (2019).

[14] Gallego S., Márquez A., Ortuño M., Francés J., Pascual I., and Beléndez A., "Diffractive and interferometric methods to characterize photopolymers with liquid crystal molecules as holographic recording material," J. European Opt. Soc. 7, 12024 (2012).

[15] Gallego S., Ortuño M., Neipp C., Márquez A., Beléndez A., Pascual I., Kelly J. V., and Sheridan J. T., "Physical and effective optical thickness of holographic diffraction gratings recorded in photopolymers," Opt. Express 13, 19391947 (2005). 